\title{
A meta-analysis of the effects of endothelial nitric oxide synthase 4ba polymorphism on renal interstitial fibrosis in diabetic nephropathy
}

\author{
Xieyi Sun ${ }^{1,2}$, Hua Gan ${ }^{3}$, Yunfeng Xia ${ }^{3}$ \\ ${ }^{1}$ Chongqing Medical University, Chongqing, China; ${ }^{2}$ Department of Nephrology, The People's Hospital of Rongchang District, Chongqing, China; \\ ${ }^{3}$ Department of Nephrology, The First Affiliated Hospital of Chongqing Medical University, Chongqing, China \\ Contributions: (I) Conception and design: X Sun; (II) Administrative support: H Gan; (III) Provision of study materials or patients: X Sun, Y Xia; (IV) \\ Collection and assembly of data: All authors; (V) Data analysis and interpretation: H Gan, Y Xia; (VI) Manuscript writing: All authors; (VII) Final \\ approval of manuscript: All authors. \\ Correspondence to: Hua Gan; Yunfeng Xia. Department of Nephrology, The First Affiliated Hospital of Chongqing Medical University, Youyi Road, \\ Yuanjiagang, Yuzhong District, Chongqing 400016, China. Email: ganhua0000@163.com; haohua11234@sina.com.
}

Background: This study aimed to investigate the relationship between endothelial nitric oxide synthase (eNOS) 4b/a gene polymorphism and renal interstitial fibrosis in patients with diabetic nephropathy (DN) via a meta-analysis, in the hope of providing guidance for the genotypic detection of DN.

Methods: The Boolean logic search method was adopted. A literature search of PubMed, Medline, CNKI, and other databases from inception to June 2020 was performed using the search terms "eNOS 4b/a", "diabetes", "renal interstitial fibrosis", and "kidney disease". Literatures with a DN group, a nonnephropathy diabetic group, and a normal control group were screened. Review Manager software was employed to perform the meta-analysis.

Results: A total of 13 articles were included in the meta-analysis, the majority of which had a low risk of bias (i.e., medium and high quality). The results of the meta-analysis indicated that the genotypic distribution frequency of eNOS4bb in patients with DN was significantly lower than that in the nonnephropathy diabetic group $(\mathrm{Z}=3.19, \mathrm{P}=0.001)$. The genotypic distribution frequency of eNOS4aa was significantly higher in non-nephropathy diabetic patients than in the normal control group $(Z=2.57, P=0.01)$. The genotypic distribution frequency of eNOS4ba was not statistically different between patients with DN and non-nephropathy diabetic patients $(\mathrm{Z}=1.45, \mathrm{P}=0.15)$. The genotypic distribution frequency of eNOS4bb in $\mathrm{DN}$ patients was significantly lower than that in the normal control group $(\mathrm{Z}=3.03, \mathrm{P}=0.002)$; however, the genotypic distribution frequency of eNOS4ba was significantly greater than that of the normal controls $(\mathrm{Z}=2.36, \mathrm{P}=0.02)$, as was that of eNOS4aa $(\mathrm{Z}=2.34, \mathrm{P}=0.02)$.

Conclusions: Genotypic polymorphism of eNOS 4b/a was closely related to DN. Moreover, the genotypic distribution frequency of eNOS4bb in DN renal interstitial patients was lower than that in non-nephropathy diabetic patients and normal controls.

Keywords: Endothelial nitric oxide synthase (eNOS 4b/a); gene polymorphism; meta-analysis; diabetic nephropathy (DN); renal interstitial fibrosis.

Submitted Dec 03, 2020. Accepted for publication Jan 22, 2021.

doi: 10.21037/apm-20-2585

View this article at: http://dx.doi.org/10.21037/apm-20-2585 


\section{Introduction}

$\mathrm{DN}$ is one of the most serious complications in diabetic patients and one of the main causes of death (1). As a leading cause of end-stage renal disease, second only to glomerulonephritis, $\mathrm{DN}$ is often accompanied by complications such as chronic hyperglycemia and proteinuria $(2,3)$. DN refers to an extremely complex metabolic disorder, and after its progression to end-stage renal disease, it is typically more challenging to treat than other kidney diseases. Therefore, early prevention and control are of great significance. Renal interstitial fibrosis is a common disease in the process of diabetic nephropathy, which can easily lead to renal insufficiency and even kidney failure (4). Its typical pathological feature is the replacement of the renal interstitium and tubules with large amounts of extracellular matrix, which is synthesized or secreted by cells including fibroblast epithelial cells and endothelial cells (5). The progression of renal interstitial fibrosis may be accompanied by changes in the expression and regulation of various cytokines, growth factors, and genes in glomerular cells. DN at the genetic level is also a prominent focus of current research (6).

Gene polymorphism refers to the simultaneous and frequent presence of 2 or more discrete variants, genotypes, or alleles in a biological population. The mechanism underlying the formation of gene polymorphisms is gene mutation, including at the whole individual, cellular, protein, and genetic levels (7). There is a variety of genetic polymorphisms including phenotypic, chromosomal, protein, enzyme, antigen, and DNA polymorphisms (8). Studies have suggested that nitric oxide (NO) can play an important regulatory role in nerve conduction, immune response, vasodilation, and blood circulation (9). Endothelial nitric oxide synthase (eNOS), as a mixed-functional oxidase, is mainly expressed in vascular endothelial cells and is also a key factor in determining the level of NO in the blood vessel wall. Literatures confirmed that eNOS had a certain influence on the progression of diabetes and the occurrence of kidney disease. The human eNOS gene is located on chromosome $7 \mathrm{q} 35-\mathrm{q} 36$, with a full length of 21-22 kb, containing 25 introns and 26 exons. Moreover, there are many genetic polymorphisms, for example, the variable number of tandem repeat polymorphisms of $27 \mathrm{bp}$ in intron 4 , including allele $\mathrm{b}$ repeated 5 times and allele a repeated 4 times, which constitute 4bb, 4aa, and 4ba multiple genotypes (10).

In summary, long-term literatures from 2003 to 2019 were innovatively collected, and different genotypic indicators were extracted to explore the relationship between eNOS 4b/a gene polymorphism and the susceptibility of DN to renal interstitial fibrosis. We present the following article in accordance with the PRISMA reporting checklist (available at http://dx.doi.org/10.21037/ apm-20-2585).

\section{Methods}

\section{Document retrieval}

The compound logic retrieval Boolean logic retrieval method was adopted to identify relevant literature for the meta-analysis. Electronic literature searches of PubMed, Medline, EMBASE, China Biomedical Literature Database, CNKI database, Wanfang Data, CQVIP database, and Google Scholar were conducted. The search terms used were "eNOS 4b/a", "diabetes", "renal interstitial fibrosis", and "kidney disease". All included literature was tracked, as were the reference lists that have published reviews, to find literature not indexed on the database. The retrieval time was from database inception to August 15, 2020. The quality of the literature was evaluated using the RevMan 5.2 software from the Cochrane Collaboration. Various search terms were combined freely; multiple searches were conducted to confirm the literatures, and the confirmed literatures were traced by the search engine. Furthermore, the experts and researchers in the corresponding field were contacted to obtain the latest research progress relating to the confirmed literatures.

\section{Literature inclusion and exclusion criteria}

The inclusion criteria were as follows: (I) the relationship between eNOS 4b/a and eNOS 4b/a in DN was investigated based on polymorphism; (II) patients in the experimental group were diabetic patients with nephropathy; (III) the control group comprised non-nephropathy diabetic patients or healthy subjects without diabetes or both; (IV) for pathological control analysis, the index comparison was reliable within the $95 \%$ confidence interval $(\mathrm{CI})$; (V) the diagnosis of DN was based on the standards of the World Health Organization; (VI) for studies without genotype or allele data, the relevant data could be obtained from the author.

Studies meeting any of the following criteria were excluded: (I) overlapping of research objects; (II) genotype 


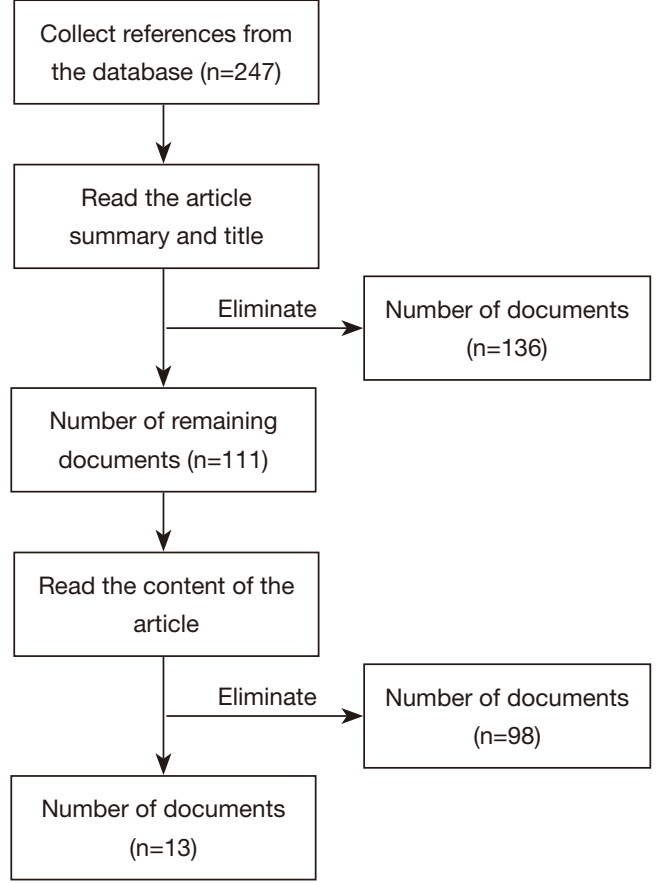

Figure 1 Schematic diagram of the literature screening process.

or allele data of DN patients containing phase 1 and phase 2 data, which could not be separated (III) genotype or allele data were unavailable, even after the original author was contacted; (IV) non-etiological studies.

Two senior experts independently screened the abstract and the full text of the articles. Three pre-experiments were conducted before the screening. If disagreement arose between the two experts, a consensus conclusion would be reached through discussion, or a third expert would be invited to arbitrate.

\section{Literature quality assessment}

The Newcastle-Ottawa Scale (NOS) of the Cochrane Collaboration was utilized to evaluate the controlled pathological studies in the meta-analysis. The NOS employs a star system (out of 9 stars) to measure the results regarding the study subjects, case comparisons, and comparisons between groups. Articles with $\geq 7$ stars were considered to be of high quality (i.e., low risk of bias); those with 2-6 stars were considered to be of medium quality (i.e., medium risk of bias); and those with $\leq 1$ star were considered to be of low quality (i.e., high risk of bias).

Two experts independently evaluated the quality of the references, and three pre-experiments were conducted before the evaluation. If disagreement arose between the two experts, a consensus conclusion would be reached through discussion, or a third expert would be invited to arbitrate.

\section{Data extraction}

Two experts independently extracted the data into a unified Excel table (Microsoft), and three pre-experiments were conducted before the extraction. If disagreement arose between the two experts, a consensus conclusion would be reached through discussion, or a third expert would be invited to arbitrate. Data extracted for the meta-analysis included: (I) first author and year of publication; (II) study population; (III) the sex, age, and body mass index (BMI) of the subjects; (IV) the disease course, type of diabetes, and diagnostic criteria of the $\mathrm{DN}$ or non-nephropathy diabetic subjects; (V) the source, sample size, genotyping ratio, and allele ratio of $\mathrm{DN}$ or diabetic control group without nephropathy or diabetes.

\section{Statistical methods}

Statistical analysis was performed using StataSE12.0 software (Stata). Odds ratios (ORs) and 95\% CIs were used to compare eNOS 4b/a gene polymorphism (eNOS4bb, eNOS4ba, and eNOS4aa) between DN patients and nonnephropathy diabetic patients, as well as between DN patients and normal controls. The bias risk evaluation chart of the Review Manager software was used to evaluate the risk of bias of the articles. Each effect was represented by the $95 \%$ CI. When $\mathrm{P}>0.1$ and $\mathrm{I}^{2}<50 \%$, the fixed-effects model was adopted for the meta-analysis; and when $\mathrm{P}<0.1$ and $\mathrm{I}^{2}>50 \%$, the random-effects model was adopted for the meta-analysis.

\section{Results}

\section{Summary of retrieved literature and NOS scale rating}

Figure 1 shows that a total of 247 articles were obtained in this search, of which 136 articles were eliminated after the abstracts and titles were read. After full-text reading of the literature, 98 articles were eliminated, and 13 articles were finally entered into the meta-analysis. The main reasons for exclusion were as follows: repetitive research subjects (26 articles), literature types other than case-control studies (62 articles), non-etiological studies (51 articles), genotype 
Table 1 Basic information of included literatures

\begin{tabular}{|c|c|c|c|c|c|c|c|c|}
\hline Author & $\begin{array}{c}\text { Year of } \\
\text { publication }\end{array}$ & $\begin{array}{l}\text { Number of } \\
\text { patients }\end{array}$ & Country & Age (years) & Male & Female & BMI $\left(\mathrm{m}^{2} / \mathrm{kg}\right)$ & $\begin{array}{c}\text { Diabetes course } \\
\text { (years) }\end{array}$ \\
\hline Santos (11) & 2011 & 376 & Brazil & $60.4 \pm 9.7$ & 215 & 161 & $28.7 \pm 5.0$ & $15.0 \pm 9.1$ \\
\hline Zheleznyakova (13) & 2014 & 253 & Russia & $62.8 \pm 8.5$ & 114 & 139 & $30.6 \pm 5.3$ & $5 \pm 0.6$ \\
\hline Shestakova (16) & 2006 & 63 & Russia & $25.7 \pm 6.5$ & 30 & 33 & $22.6 \pm 2.4$ & $12.6 \pm 2.8$ \\
\hline Dong (17) & 2005 & 70 & China & $63.1 \pm 7.3$ & 26 & 44 & / & $15.3 \pm 3.1$ \\
\hline Algenabi (18) & 2019 & 97 & Iraq & - & - & - & - & - \\
\hline Ahluwalia (22) & 2008 & 195 & India & $60.0 \pm 6.2$ & 68 & 127 & $28.0 \pm 4.0$ & $16.5 \pm 6.4$ \\
\hline Luo (23) & 2003 & 49 & China & $59.0 \pm 10.5$ & 29 & 20 & $22.4 \pm 3.0$ & $9.9 \pm 6.4$ \\
\hline
\end{tabular}

BMI, body mass index.

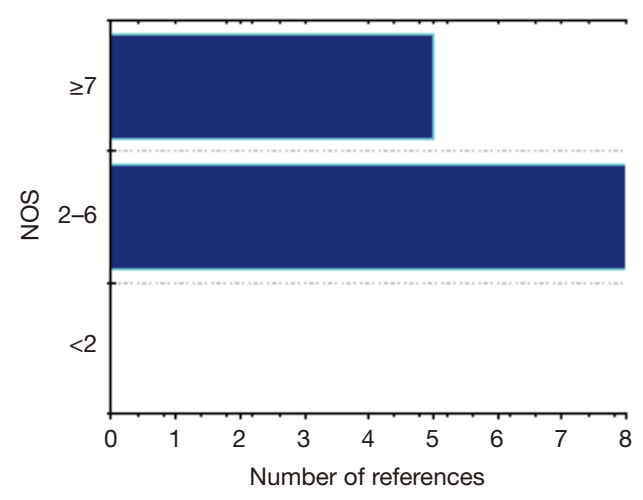

Figure 2 Quality rating results of the NOS scale. NOS, Newcastle-Ottawa Scale.

or allele data containing DN stages 1 and 2 that could not be separated (49 articles), and research-related information unable to be extracted (59 studies).

Table 1 displays the basic information of the included studies. The time interval was 2000-2019, and the studies came from Europe, Asia, Africa, and other regions. The average BMI of the research subjects of all literatures was $27 \mathrm{~m}^{2} / \mathrm{kg}$, and the average disease course was 13 years. Figure 2 presents the NOS scale scores of the articles. There were 5 articles with $\geq 7$ stars, 8 articles with 2-6 stars, and no articles with $\leq 1$ star, meaning all articles included in the present meta-analysis were of medium to high quality.

\section{Results of risk bias evaluation of the articles}

Figure 3 and Figure 4 depict the results of multiple risk bias evaluations of the articles performed using the Review Manager software. All methodological features of the articles were included, and the evaluation results were input into the software to generate a bias risk evaluation chart. For the included articles, it was obvious that biases of random sequence generation (selection bias), allocation concealment (selection bias), blinding method of result evaluation (measurement bias), incomplete result data (follow-up bias), and selective reporting (reporting bias) were all at low risk. Moreover, the low-risk bias evaluation results of the blinding method of subjects and researchers (implementation bias) and other biases were around 50\%. Except for the research of Luo et al. [2003], the articles had an obvious low risk of bias.

\section{Comparison of eNOS4bb genotype distribution between DN patients and non-nephropathy diabetic patients}

Figure 5 shows the comparison of eNOS4bb genotype distribution between DN patients and non-nephropathy 


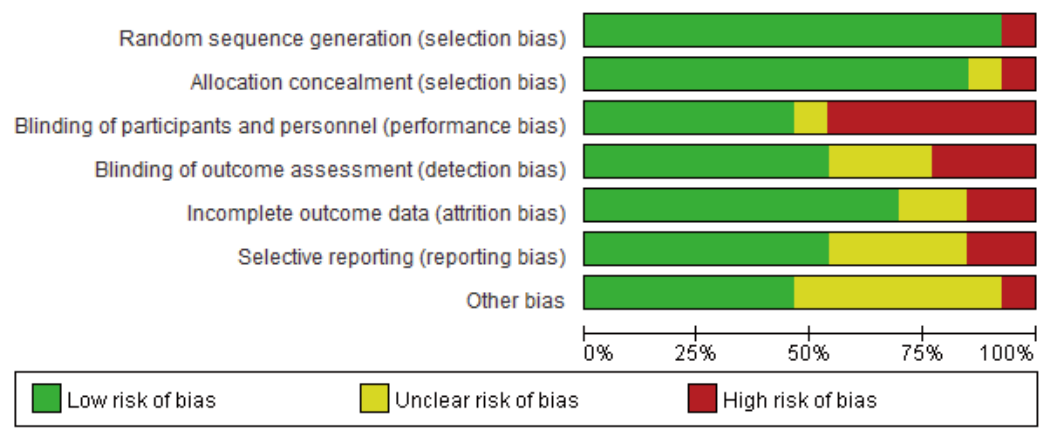

Figure 3 Evaluation results of the risk bias of the literatures.

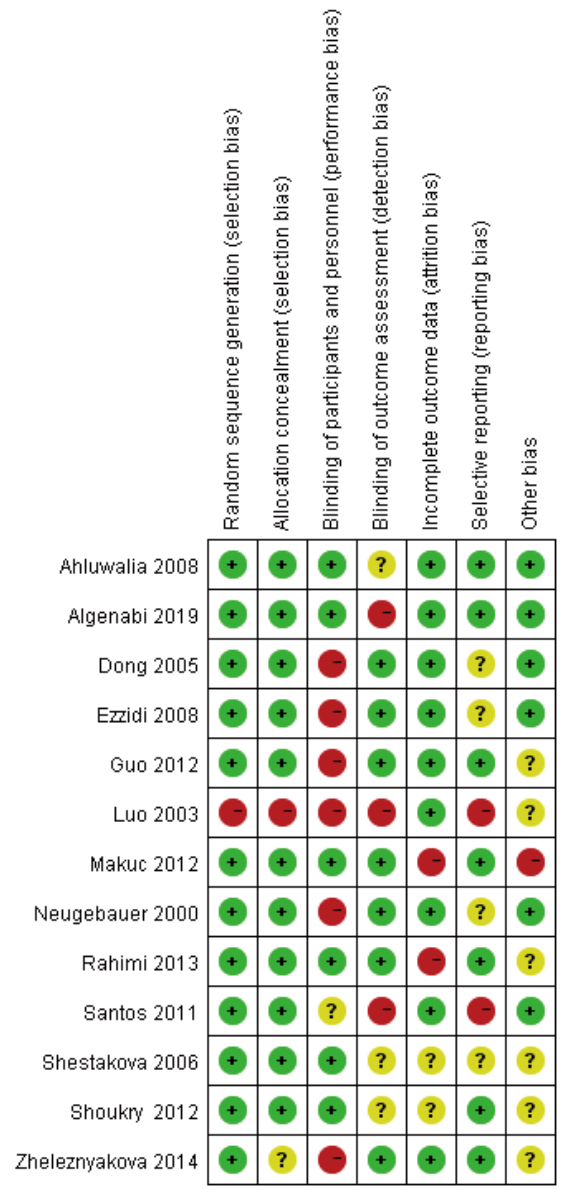

Figure 4 Evaluation results of multiple studies in the literature corresponding to multiple risk biases.

diabetic patients. Figure $5 A$ relates to $\mathrm{DN}$ patients, and Figure $5 B$ relates to non-nephropathy diabetic patients. Ezzidi et al.'s study accounted for the largest proportion $(10.5 \%)$ of the final combined results, followed by the studies of Santos et al. (9.9\%) and Ahluwalia et al. (9.6\%). Further, the horizontal lines of the $95 \% \mathrm{CI}$ of most studies were on the left side of the invalid vertical line, and some horizontal lines crossed the invalid vertical line. The horizontal line of the $95 \%$ CI was on the right of the invalid vertical line.

Among the 13 included studies, 1,919 subjects were included in group A and 1,970 subjects in group B. The distribution of eNOS4bb genotypes in the 2 groups was heterogeneous $\left(\chi^{2}=36.87, \mathrm{I}^{2}=67 \%, \mathrm{P}=0.0002\right)$, and the combined effect size (diamond block) was on the left side of the invalid line (OR: $-0.09,95 \%$ CI: $-0.14,-0.03$ ). Random-effects model analysis showed that the genotypic distribution frequency of eNOS4bb in group A was significantly lower than that in group $\mathrm{B}(\mathrm{Z}=3.19, \mathrm{P}=0.001)$.

Figure 6 displays a funnel plot showing eNOS4bb genotype distribution in DN patients and non-nephropathy diabetic patients. As can clearly be seen, the number of circles on either side of the midline is similar, and the circles are basically concentrated on the midline, indicating high research accuracy and no publication bias.

\section{Comparison of eNOS4ba genotype distribution between DN patients and non-nephropathy diabetic patients}

Figure 7 shows a comparison of eNOS4ba genotype distribution between DN patients and non-nephropathy diabetic patients. Figure $7 A$ relates to $\mathrm{DN}$ patients, and Figure $7 B$ relates to non-nephropathy diabetic patients. Ezzidi et al.'s study accounted for the largest proportion of the final combined results (10.0\%), followed by the studies of Santos et al. (9.5\%) and Ahluwalia et al. (9.5\%). For most studies, the horizontal line of the $95 \%$ CI crossed the invalid vertical line. For a few studies, the horizontal line of the $95 \% \mathrm{CI}$ was on the right of the invalid vertical line. For no studies was the horizontal line of the $95 \% \mathrm{CI}$ on the left 


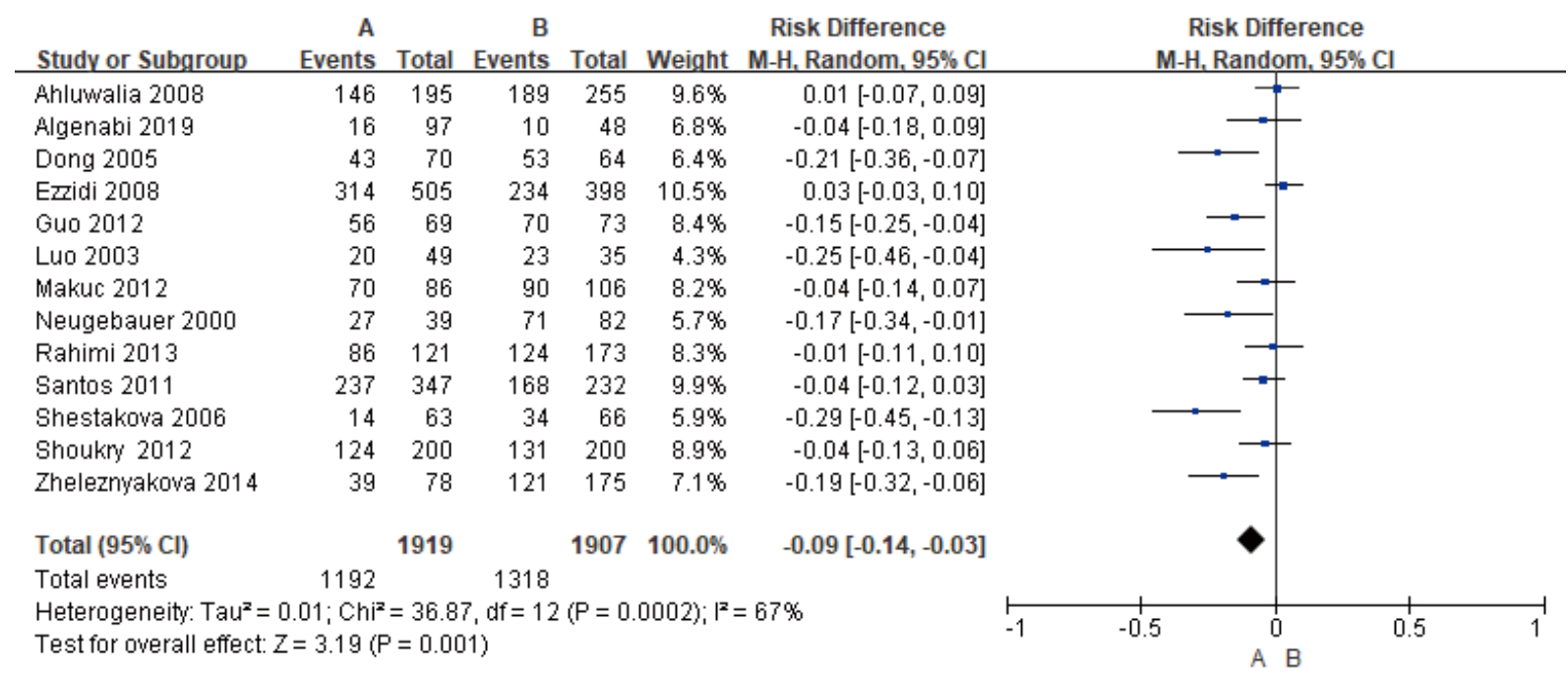

Figure 5 Comparison of eNOS4bb genotype distribution between DN patients and non-nephropathy patients. A was diabetes and renal interstitial patients, B was diabetic non-nephropathy patients. DN, diabetic nephropathy.

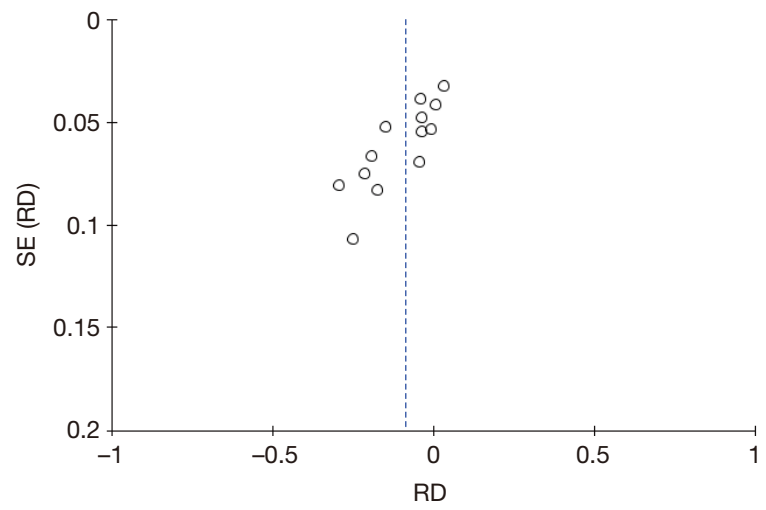

Figure 6 Funnel plots of eNOS4bb genotype distribution in DN patients and non-nephropathy patients. SE $[\log (\mathrm{OR})]$ is the standard error; OR is the effect size. DN, diabetic nephropathy.

of the invalid vertical line.

Among the 13 included studies, 1,919 subjects were included in group A and 1,970 subjects in group B. The distribution of eNOS4ba genotypes in the 2 groups was heterogeneous $\left(\chi^{2}=41.36, \mathrm{I}^{2}=71 \%, \mathrm{P}<0.0001\right)$, and the combined effect size (diamond-shaped block) crossed the invalid line (OR value: $0.04,95 \%$ CI: $-0.01,0.09$ ). Random-effects model analysis showed that the genotypic distribution frequency of eNOS4ba between group A and group B was not statistically different $(\mathrm{Z}=1.45, \mathrm{P}=0.15)$.

Figure 8 shows the funnel plot of eNOS4ba genotype distribution in DN patients and non-nephropathy diabetic patients. The circles of the included studies are roughly symmetrically distributed on both sides of the midline, and most of them are concentrated on the midline, showing no publication bias in the included literatures.

\section{Comparison of eNOS4aa genotype distribution between DN patients and non-nephropathy diabetic patients}

Figure 9 shows a comparison of eNOS4ba genotype distribution between DN patients and non-nephropathy diabetic patients. Figure $9 A$ relates to DN patients, and Figure $9 B$ relates to non-nephropathy diabetic patients. Santos et al.'s study accounted for the largest proportion of the final combined results $(11.8 \%)$, followed by the studies of Ezzidi et al. [2008] (11.3\%) and Dong et al. [2005] (11.5\%). The horizontal lines of the $95 \%$ CIs of most studies crossed the invalid vertical line, and the horizontal lines of the $95 \%$ CIs of a few studies were on the right of the invalid vertical line. For no studies was the horizontal line of the $95 \%$ CI on the left of the invalid vertical line.

Among the 13 studies included, group A included a total of 1,919 subjects, and group B included a total of 1907 subjects. The distribution of eNOS4ba genotypes in the 2 groups was heterogeneous $\left(\chi^{2}=34.83, \mathrm{I}^{2}=66 \%, \mathrm{P}=0.0005\right)$, and the combined effect size (diamond-shaped block) crossed the invalid line (OR: 0.03; 95\% CI: 0.01, 0.05). Random-effects model analysis showed that the genotypic distribution frequency of eNOS4aa in group A was 


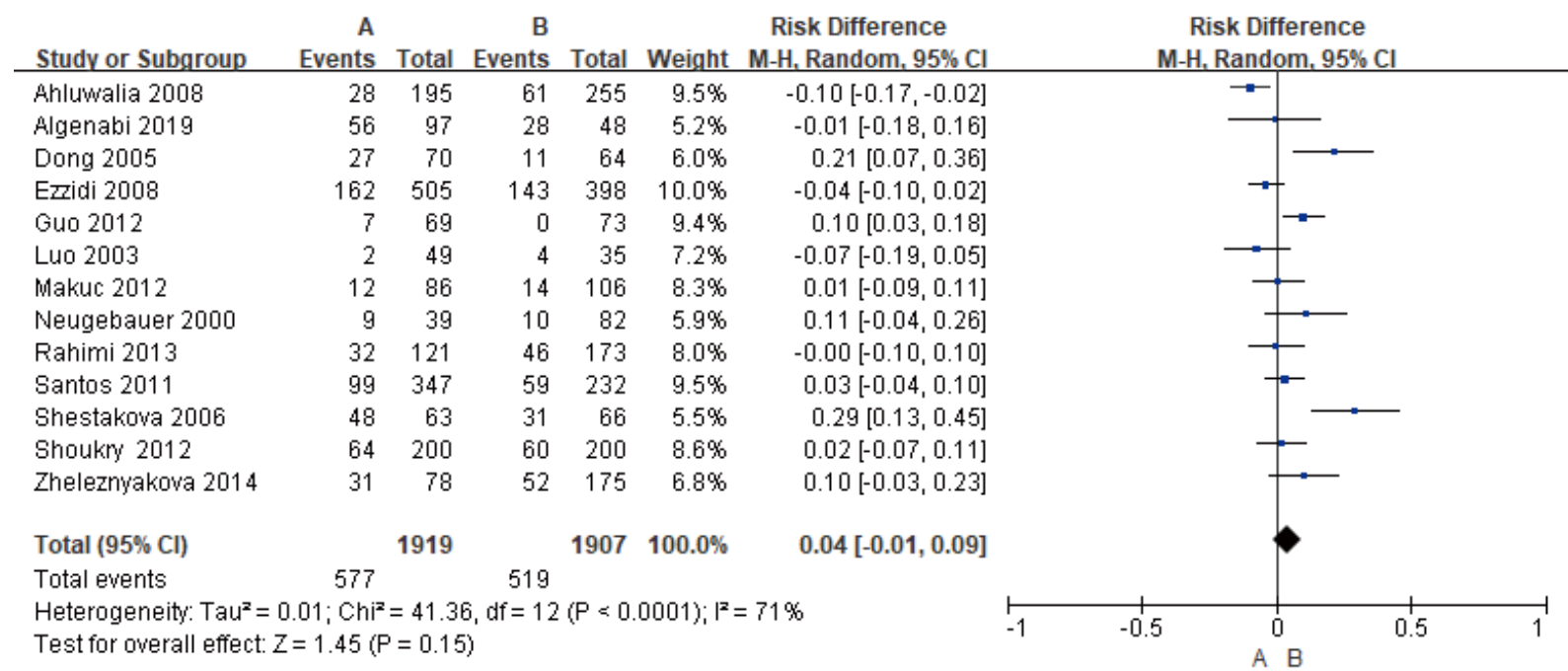

Figure 7 Comparison of eNOS4ba genotype distribution between DN patients and non-nephropathy patients. A was diabetes and renal interstitial patients, B was diabetic non-nephropathy patients. DN, diabetic nephropathy.

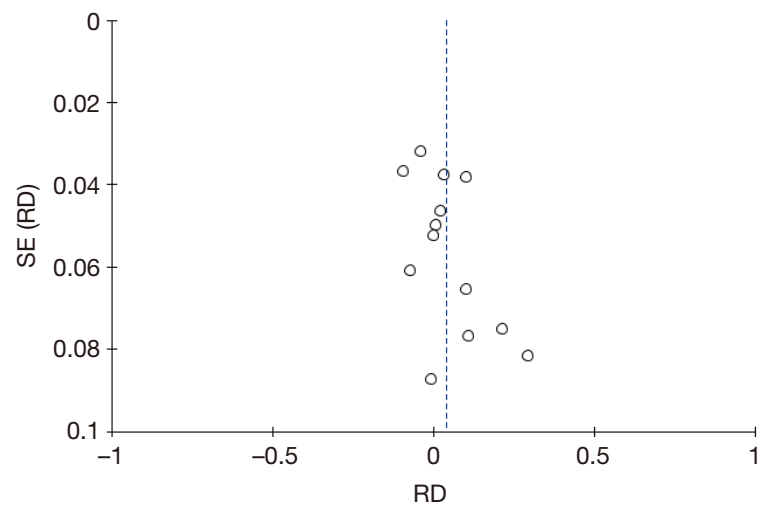

Figure 8 Funnel plots of eNOS4ba genotype distribution in DN patients and non-nephropathy patients. SE (RD) is the standard error; RD is the effect size. DN, diabetic nephropathy.

significantly higher than that in group $\mathrm{B}(\mathrm{Z}=2.57, \mathrm{P}=0.01)$.

Figure 10 shows a funnel plot of the eNOS4aa genotype distribution of DN patients and non-nephropathy diabetic patients. Most of the circles of the included studies are concentrated on the midline, showing that the accuracy of the studies was relatively high. Therefore, the included studies had no publication bias.

\section{Comparison of eNOS4bb genotype distribution between DN patients and normal controls}

Figure 11 shows a comparison of the genotype distribution of eNOS4bb between DN patients and normal controls. Figure $11 \mathrm{~A}$ relates to DN patients, and Figure $11 \mathrm{C}$ relates to normal controls. The results of Ezzidi et al. accounted for the largest proportion (18.4\%) of the final combined results, followed by the results of Santos et al. (15.9\%). The horizontal lines of the $95 \%$ CIs of most of the studies were on the left of the invalid vertical line, and the horizontal lines of the $95 \%$ CIs of a few studies crossed the invalid vertical line. For no studies was the horizontal line of the 95\% CI on the right of the invalid vertical line.

Among the 8 studies included in this comparison, 1,297 subjects were included in group A and 1,339 subjects were included in group C. The distribution of eNOS4bb genotypes between the 2 groups was heterogeneous $\left(\chi^{2}=27.29, I^{2}=74 \%\right.$, $\mathrm{P}=0.0003$ ), and the combined effect size (diamond block) was on the left side of the invalid line (OR: 0.67 , and 95\% CI: $0.56,0.80)$. Random-effects model analysis showed that the genotypic distribution frequency of eNOS4bb in group A was significantly lower than that in group $\mathrm{C}(\mathrm{Z}=3.03, \mathrm{P}=0.002)$.

Figure 12 is a funnel plot showing eNOS4bb genotype distribution of DN patients and normal controls. The circles of the included studies have an asymmetrical distribution, indicating the presence of publication bias.

\section{Comparison of eNOS4ba genotype distribution between DN patients and normal controls}

Figure 13 shows a comparison of the genotype distribution 


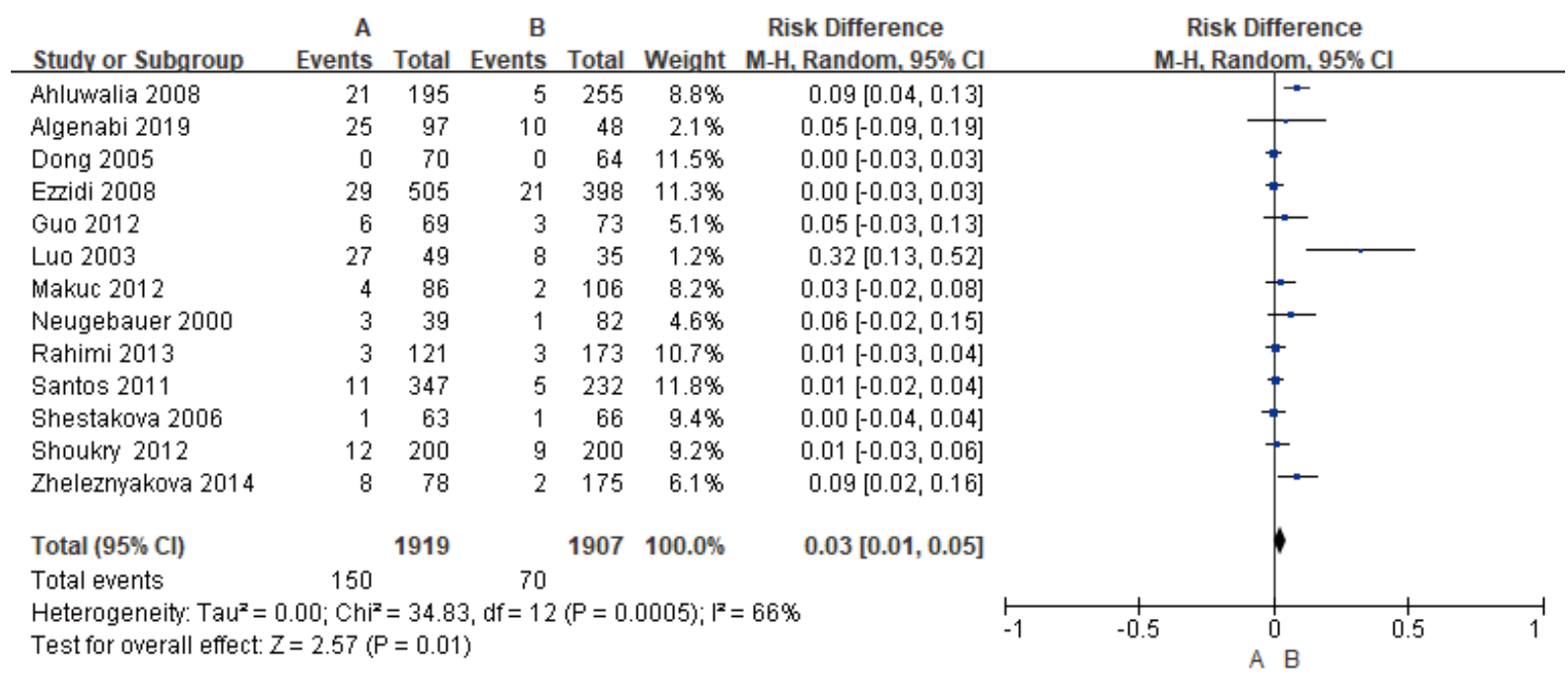

Figure 9 Comparison of eNOS4aa genotype distribution between DN patients and non-nephropathy patients. A was diabetes and renal interstitial patients, B was diabetic non-nephropathy patients. DN, diabetic nephropathy.

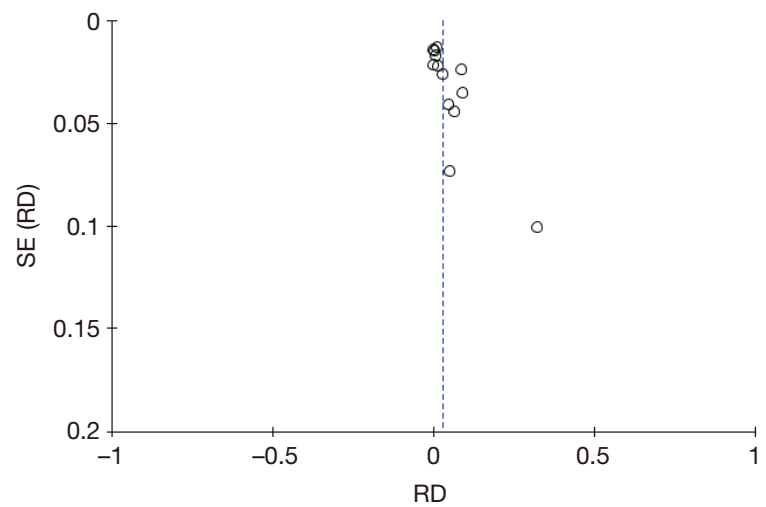

Figure 10 Funnel plots of eNOS4aa genotype distribution in DN patients and non-nephropathy patients. SE (RD) is the standard error; RD is the effect size. DN, diabetic nephropathy.

of eNOS4ba between DN patients and normal controls. Figure $13 \mathrm{~A}$ relates to DN patients, and Figure $13 C$ relates to normal controls. Ezzidi et al.'s study accounted for the largest proportion of the final combined results (57.8\%), followed by the studies of Santos et al. (16.2\%) and Rahimi et al. (10.9\%). Furthermore, the horizontal lines of the $95 \%$ CIs of most studies crossed the invalid vertical line, and the horizontal lines of the $95 \%$ CIs of a few studies were on the right of the invalid vertical line. For no studies was the horizontal line of the $95 \% \mathrm{CI}$ on the left of the invalid vertical line.

Among the 8 included studies, 1297 subjects were included in group A, and 1339 subjects were included in group C. No heterogeneity was found in the distribution of eNOS4ba genotype between the 2 groups $\left(\chi^{2}=10.45\right.$, $\mathrm{I}^{2}=33 \%, \mathrm{P}=0.16$ ), and the combined effect size (diamond block) was on the right side of the invalid line (OR: 1.25, 95\% CI: 1.04, 1.50). The fixed-effects model showed that the genotypic distribution frequency of eNOS4ba in group A was significantly higher than that in group $C(Z=2.36$, $\mathrm{P}=0.02)$.

Figure 14 depicts a funnel plot showing the eNOS4ba genotype distribution of $\mathrm{DN}$ patients and normal controls. The circles of the included studies are concentrated in the top area, indicating high research accuracy, and they are distributed on both sides of the midline, in a roughly symmetrical form, reflecting an absence of publication bias.

\section{Comparison of eNOS4aa genotype distribution between DN patients and normal controls}

Figure 15 shows a comparison of the genotype distribution of eNOS4aa between DN patients and normal controls. Figure $15 \mathrm{~A}$ relates to DN patients, and Figure $15 \mathrm{C}$ relates to normal controls. Ezzidi et al.'s study accounted for the largest proportion of the final combined results $(22.2 \%)$, followed by the studies of Algenabi et al. (20.3\%) and Santos et al. (15.3\%). Furthermore, for some studies, the horizontal line of the $95 \%$ CI crossed the invalid vertical line. For some studies, the horizontal line of the $95 \%$ CI 


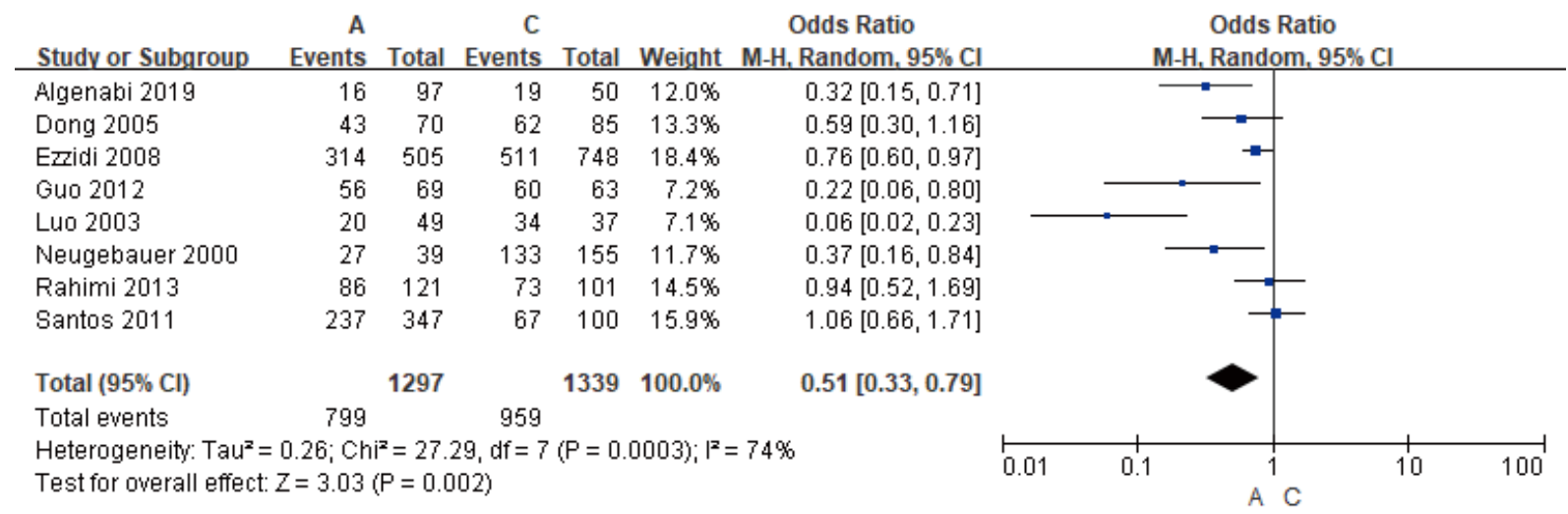

Figure 11 Comparison of eNOS4bb genotype distribution between DN patients and normal controls. A was diabetes and renal interstitial patients, C was normal controls. DN, diabetic nephropathy.

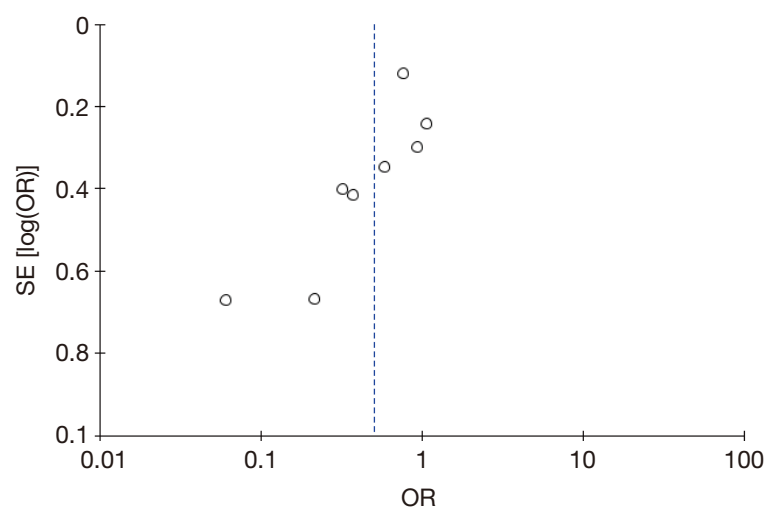

Figure 12 Funnel plots of eNOS4bb genotype distribution in DN patients and normal controls. SE $[\log (\mathrm{OR})]$ is the standard error; OR is the effect size. DN, diabetic nephropathy. was on the right of the invalid vertical line. For no studies was the horizontal line of the $95 \%$ CI on the left of the invalid vertical line.

Among the 8 included studies, 1297 subjects were included in group A, and 1339 subjects were included in group C. The distribution of eNOS4ba genotypes in the 2 groups was heterogeneous $\left(\chi^{2}=18.64, \mathrm{I}^{2}=62 \%, \mathrm{P}=0.009\right)$, and the combined effect size (diamond block) was on the right side of the invalid line (OR: 2.64, 95\% CI: 1.17, 5.96). Random-effects model analysis showed that the genotypic distribution frequency of eNOS4aa in group A was significantly higher than that in group $C(Z=2.34, P=0.02)$.

Figure 16 depicts a funnel plot showing eNOS4aa genotype distribution of DN patients and normal controls.

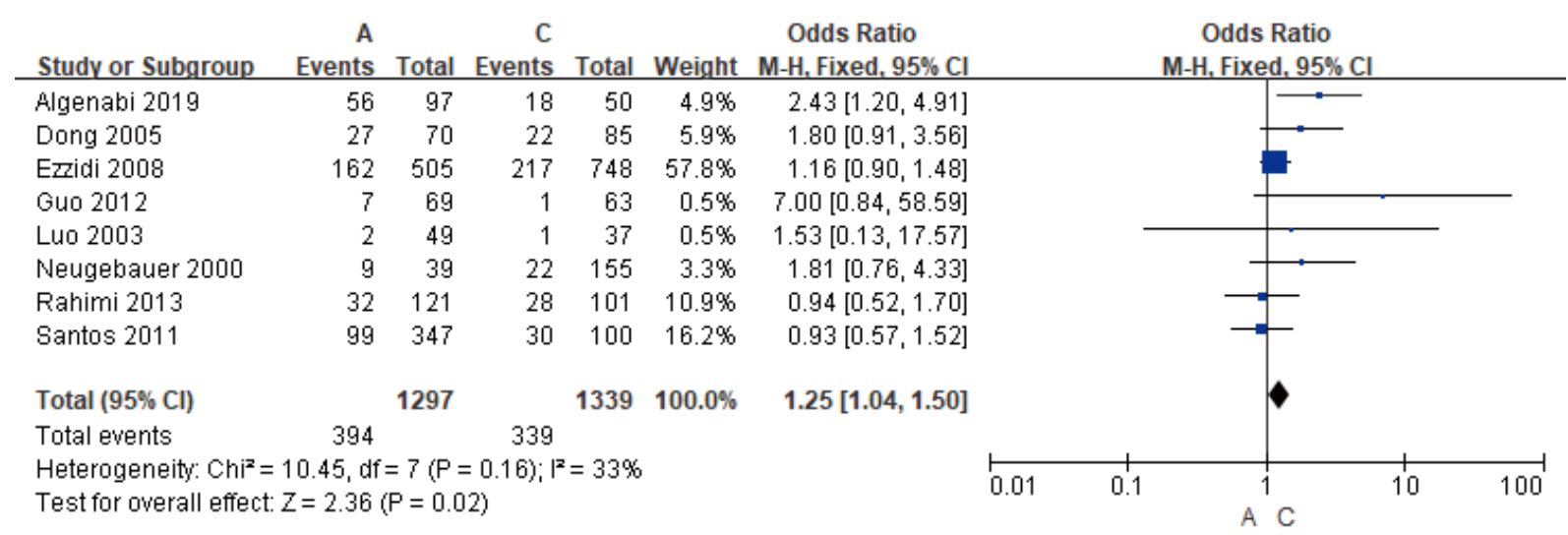

Figure 13 Comparison of eNOS4ba genotype distribution between DN patients and normal controls. A was diabetes and renal interstitial patients, C was normal controls. DN, diabetic nephropathy. 


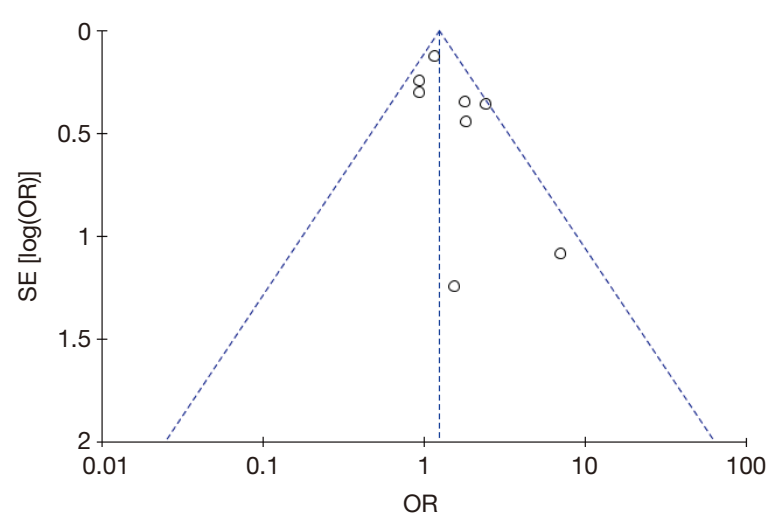

Figure 14 Funnel plots of eNOS4ba genotype distribution in DN patients and normal controls. SE $[\log (\mathrm{OR})]$ is the standard error; OR is the effect size. DN, diabetic nephropathy.
The circles of the included studies are distributed on both sides of the midline, and the funnel plot is roughly symmetrical, indicating the absence of publication bias.

\section{Discussion}

$\mathrm{DN}$ is one of the most dangerous microvascular complications in diabetic patients, with a high probability of occurrence. Clinical observations have shown that the incidence of nephropathy in patients with type 1 and type 2 diabetes was approximately $35 \%$ and $25 \%$, respectively (24). High blood pressure, hyperlipidemia, and obesity greatly increase the risk of renal interstitial fibrosis. However, the specific mechanisms of the occurrence of renal interstitial

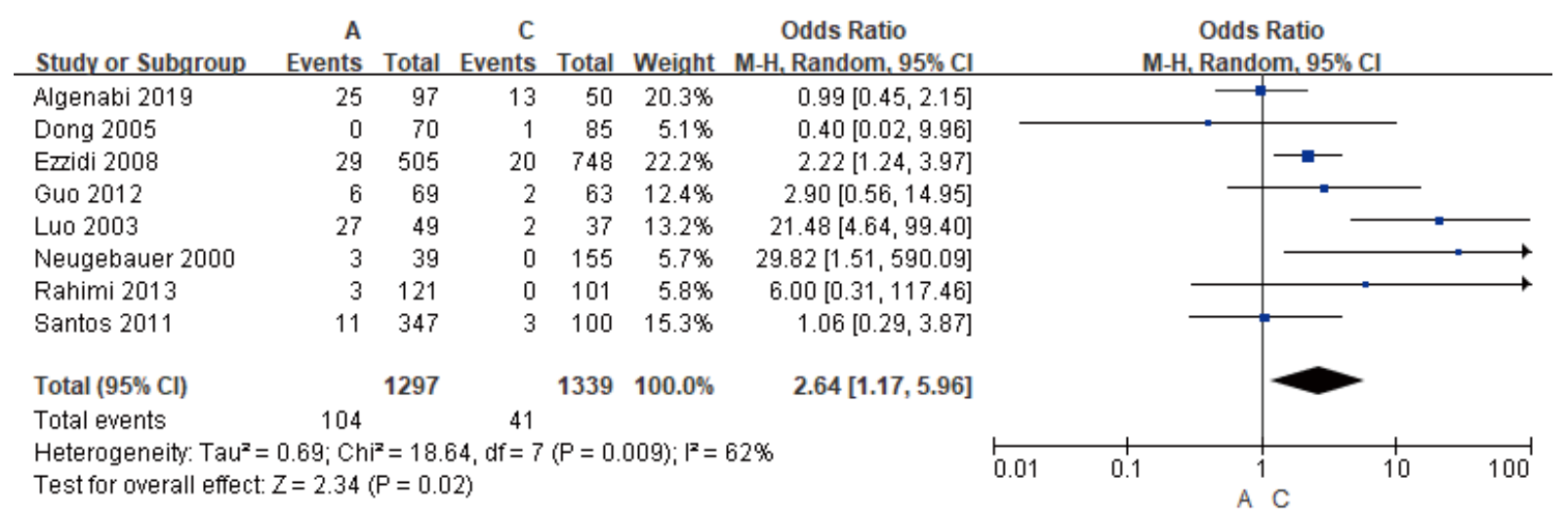

Figure 15 Comparison of eNOS4aa genotype distribution between DN patients and normal controls. A was diabetes and renal interstitial patients, C was normal controls. DN, diabetic nephropathy.

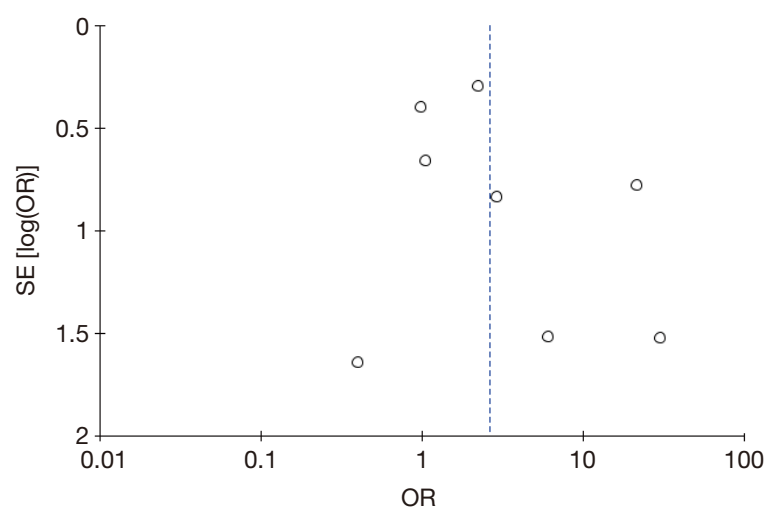

Figure 16 Funnel plots of eNOS4aa genotype distribution in DN patients and normal controls. SE $[\log (\mathrm{OR})]$ is the standard error; OR is the effect size. DN, diabetic nephropathy. fibrosis and the progression of uremia are not entirely clear (25). Relevant studies have revealed that vascular endothelial dysfunction caused by NOS damage is a key factor for DN (26). Therefore, compound logic retrieval Boolean logic retrieval method was employed, and a metaanalysis was implemented of 13 studies involving DN patients as the experimental group and non-nephropathy diabetic patients or healthy individuals as controls. We aimed to explore the relationship between eNOS 4 ba gene polymorphism and the occurrence of renal interstitial fibrosis in $\mathrm{DN}$, in the hope of providing guidance for the gene-level detection of renal interstitial fibrosis in patients with DN (27).

The results showed that the eNOS4bb genotype distribution of DN patients and diabetic non-nephropathy 
diabetic patients was heterogeneous $\left(\chi^{2}=36.87, \mathrm{I}^{2}=67 \%\right.$, $\mathrm{P}=0.0002)$. Moreover, the frequency of the eNOS4bb genotype distribution in patients with $\mathrm{DN}$ was significantly lower than that in non-nephropathy diabetic patients ( $\mathrm{Z}=3.19, \mathrm{P}=0.001$ ), suggesting that the occurrence of renal interstitial fibrosis in diabetic patients was related to the eNOS4bb genotype (28). The eNOS4bb genotype decreased with the progression of renal interstitium. There was no evident difference in the frequency of eNOS4ba genotype distribution between DN patients and nonnephropathy diabetic patients $(\mathrm{Z}=1.45, \mathrm{P}=0.15)$, suggesting that the distribution of the eNOS4ba genotype had no association with the process of renal interstitial fibrosis in DN (29). The frequency of eNOS4aa genotype distribution in DN renal interstitial patients was significantly greater than that in non-nephropathy diabetic patients $(Z=2.57$, $\mathrm{P}=0.01$ ), indicating that the eNOS4aa genotype increased with the progression of renal interstitial fibrosis.

From the comparison between DN patients and normal controls, the distribution of the eNOS4bb genotype between DN patients and normal controls was heterogeneous $\left(\chi^{2}=27.29, \mathrm{I}^{2}=74 \%, \mathrm{P}=0.0003\right)$. Moreover, the genotypic distribution frequency of eNOS4bb in DN patients was significantly lower than that in the normal controls $(\mathrm{Z}=3.03, \mathrm{P}=0.002)$, which was consistent with the above results, indicating that the eNOS4bb genotype plays a role in the development of $\mathrm{DN}$ and that it decreases with the development of kidney disease (30). No heterogeneity was found in the distribution of the eNOS4ba genotype between DN patients and normal controls $\left(\chi^{2}=10.45\right.$, $\mathrm{I}^{2}=33 \%, \mathrm{P}=0.16$ ), while its distribution in $\mathrm{DN}$ patients was significantly higher than that in normal controls $(\mathrm{Z}=2.36$, $\mathrm{P}=0.02)$. This result indicated that with the development of nephropathy in diabetic patients, the genotype of eNOS4ba showed an increasing trend. Therefore, the eNOS4ba genotype was found to play an important role in the process of DN. The greater the frequency of eNOS4ba genotype distribution, the higher the probability of DN (31). The genotypic distribution frequency of eNOS4ba in DN patients was significantly greater than that in normal controls $(\mathrm{Z}=2.34, \mathrm{P}=0.02)$, which showed that the eNOS4aa genotype has a greater impact on DN.

\section{Conclusions}

In this study, the relationship between eNOS 4b/a genetic polymorphism and the occurrence of $\mathrm{DN}$ was explored via a meta-analysis. However, the meta-analysis has limitations due to the influence of various confounding factors. The articles selected for analysis were all case-control studies, which introduced a survival bias. In addition, the survival time of patients with DN is relatively short, and many patients carrying risk genes may not have been included in the studies, thus greatly reducing the combined effect size. Follow-up analysis of diabetic patients should be carried out in the future to explore the occurrence of renal interstitial fibrosis in diabetic patients, so as to further the results of the meta-analysis. In summary, eNOS 4b/a genotype polymorphism is closely associated with $\mathrm{DN}$.

\section{Acknowledgments}

Funding: None.

\section{Footnote}

Reporting Checklist: The authors have completed the PRISMA reporting checklist. Available at http://dx.doi. org/10.21037/apm-20-2585

Conflicts of Interest: All authors have completed the ICMJE uniform disclosure form (available at http://dx.doi. org/10.21037/apm-20-2585). The authors have no conflicts of interest to declare.

Ethical Statement: The authors are accountable for all aspects of the work in ensuring that questions related to the accuracy or integrity of any part of the work are appropriately investigated and resolved.

Open Access Statement: This is an Open Access article distributed in accordance with the Creative Commons Attribution-NonCommercial-NoDerivs 4.0 International License (CC BY-NC-ND 4.0), which permits the noncommercial replication and distribution of the article with the strict proviso that no changes or edits are made and the original work is properly cited (including links to both the formal publication through the relevant DOI and the license). See: https://creativecommons.org/licenses/by-nc-nd/4.0/.

\section{References}

1. Pawlik A, Błaszczyk H, Rać M, et al. NOS3 Gene rs1799983 and rs2070744 Polymorphisms in Patients with Unstable Angina. J Vasc Res 2020;57:136-42.

2. Garme Y, Saravani R, Galavi HR. Association of nitric 
oxide synthase 3 gene polymorphism with the risk of type 2 diabetes. Biomed Rep 2017;7:85-9.

3. Nasr HB, Dimassi S, M'hadhbi R, et al. Functional G894T (rs1799983) polymorphism and intron-4 VNTR variant of nitric oxide synthase (NOS3) gene are susceptibility biomarkers of obesity among Tunisians. Obes Res Clin Pract 2016;10:465-75.

4. Tashiro Y, Han Q, Tan Y, et al. Oral Recombinant Methioninase Inhibits Diabetes Onset in Mice on a Highfat Diet. In Vivo 2020;34:973-8.

5. Menter T, Haslbauer JD, Nienhold R, et al. Postmortem examination of COVID-19 patients reveals diffuse alveolar damage with severe capillary congestion and variegated findings in lungs and other organs suggesting vascular dysfunction. Histopathology 2020;77:198-209.

6. Fan Y, Fan H, Zhu B, et al. Astragaloside IV protects against diabetic nephropathy via activating eNOS in streptozotocin diabetes-induced rats. BMC Complement Altern Med 2019;19:355.

7. King GL, Park K, Li Q. Selective Insulin Resistance and the Development of Cardiovascular Diseases in Diabetes: The 2015 Edwin Bierman Award Lecture. Diabetes 2016;65:1462-71.

8. Chen Z, D S Oliveira S, Zimnicka AM, et al. Reciprocal regulation of eNOS and caveolin-1 functions in endothelial cells. Mol Biol Cell 2018;29:1190-202.

9. Qi W, Keenan HA, Li Q, et al. Pyruvate kinase M2 activation may protect against the progression of diabetic glomerular pathology and mitochondrial dysfunction. Nat Med 2017;23:753-62.

10. Denimal D, Monier S, Brindisi MC, et al. Impairment of the Ability of HDL From Patients With Metabolic Syndrome but Without Diabetes Mellitus to Activate eNOS: Correction by S1P Enrichment. Arterioscler Thromb Vasc Biol 2017;37:804-11.

11. Santos KG, Crispim D, Canani LH, et al. Association of eNOS gene polymorphisms with renal disease in Caucasians with type 2 diabetes. Diabetes Res Clin Pract 2011;91:353-62.

12. Makuc J, Petrovic D. No association between NOS2 and NOS3 polymorphisms and diabetic nephropathy in type 2 diabetics. Cent Eur J Biol 2012;7:404-10.

13. Zheleznyakova AV, Lebedeva NO, Vikulova OK, et al. Risk of chronic kidney disease in type 2 diabetes determined by polymorphisms in NOS3, APOB, KCNJ11, TCF7L2 genes as compound effect of risk genotypes combination. Diabetes Mellitus 2014;17:23-30.

14. Rahimi Z, Rahimi Z, Shahvaisi-Zadeh F, et al. eNOS 4a/ b polymorphism and its interaction with eNOS G894T variants in type 2 diabetes mellitus: modifying the risk of diabetic nephropathy. Dis Markers 2013;34:437-43.

15. Shoukry A, Shalaby SM, Abdelazim S, et al. Endothelial nitric oxide synthase gene polymorphisms and the risk of diabetic nephropathy in type 2 diabetes mellitus. Genet Test Mol Biomarkers 2012;16:574-9.

16. Shestakova MV, Vikulova OK, Gorashko NM, et al. The relationship between genetic and haemodynamic factors in diabetic nephropathy (DN): Case-control study in type 1 diabetes mellitus (T1DM). Diabetes Res Clin Pract 2006;74:S41-S50.

17. Dong YH, Qu SP, Lü WS, et al. Gene polymorphism in chromosome 7q35 and susceptibility to diabetic nephropathy. Chinese journal of diabetes 2005;21:47-50.

18. Algenabi AHA, Al-Janabi LM, Mohammed AJ. Endothelial Nitric Oxide Synthase (Enos) 4b/A Gene Polymorphism And The Risk Of Diabetic Nephropathy In Type 2 Diabetes. University of Thi-Qar Journal Of Medicine 2019;18:44-53.

19. Guo XJ, Liu SJ. The association between endothelial nitric oxide synthase gene polymorphism and type 2 diabetic nephropathy. China Healthcare Innovation 2012;6:3-5.

20. Neugebauer S, Baba T, Watanabe T. Association of the nitric oxide synthase gene polymorphism with an increased risk for progression to diabetic nephropathy in type 2 diabetes. Diabetes 2000;49:500-3.

21. Ezzidi I, Mtiraoui N, Mohamed MB, et al. Association of endothelial nitric oxide synthase Glu298Asp, 4b/a, and $-786 \mathrm{~T}>\mathrm{C}$ gene variants with diabetic nephropathy. J Diabetes Complications 2008;22:331-8.

22. Ahluwalia TS, Ahuja M, Rai TS, et al. Endothelial nitric oxide synthase gene haplotypes and diabetic nephropathy among Asian Indians. Mol Cell Biochem 2008;314:9-17.

23. Luo H, Ning YY. Association of polymorphism of endothelialnitricoxide synthase gene with diabetic nephropathy. Chinese Journal Of Diabetes Mellitus 2003;11:317-20.

24. Chen F, Li YM, Yang LQ, et al. Association of NOS2 and NOS3 gene polymorphisms with susceptibility to type 2 diabetes mellitus and diabetic nephropathy in the Chinese Han population. IUBMB Life 2016;68:516-25.

25. Gunawan A, Fajar J K, Tamara F, et al. Nitride oxide synthase 3 and klotho gene polymorphisms in the pathogenesis of chronic kidney disease and age-related cognitive impairment: a systematic review and metaanalysis. F1000Research 2020;9:252.

26. Shamsaldeen YA, Lione LA, Benham CD. Dysregulation 
of TRPV4, eNOS and caveolin-1 contribute to endothelial dysfunction in the streptozotocin rat model of diabetes.

Eur J Pharmacol 2020;888:173441.

27. Sadati SM, Radfar M, Hamidi AK, et al. Association Between the Polymorphism of Glu298Asp in Exon 7 of the eNOS Gene With Foot Ulcer and Oxidative Stress in Adult Patients With Type 2 Diabetes. Can J Diabetes 2018;42:18-22.

28. Mirfeizi M, Hasanzad M, Sattari M, et al. Association of eNOS and ACE gene polymorphisms as a genetic risk factor in gestational diabetes in Iranian women. J Diabetes Metab Disord 2018;17:123-7.

29. Oltulu F, Buhur A, Gürel Ç, et al. Mid-dose losartan mitigates diabetes-induced hepatic damage by regulating

Cite this article as: Sun X, Gan H, Xia Y. A metaanalysis of the effects of endothelial nitric oxide synthase 4ba polymorphism on renal interstitial fibrosis in diabetic nephropathy. Ann Palliat Med 2021;10(1):633-645. doi: 10.21037/apm-20-2585

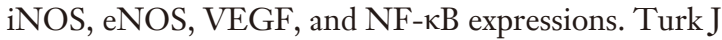
Med Sci 2019;49:1582-9.

30. Rizvi S, Raza ST, Rahman Q, et al. Association of endothelial nitric oxide synthase (eNOS) and norepinephrine transporter (NET) genes polymorphism with type 2 diabetes mellitus. Mol Biol Rep 2019;46:5433-41.

31. Idris-Khodja N, Ouerd S, Mian MOR, et al. Endothelin-1 Overexpression Exaggerates Diabetes-Induced Endothelial Dysfunction by Altering Oxidative Stress. Am J Hypertens 2016;29:1245-51.

(English Language Editor: J. Reynolds) 